\title{
GENERACIÓN DE BANDAS DE FRECUENCIAS PERMITIDAS EN LÍNEAS DE TRANSMISIÓN DUAL CON CORRELACIÓN DE LARGO ALCANCE EN EL DESORDEN
}

\section{GENERATION OF ALLOWED FREQUENCY BANDS IN DUAL TRANSMISSION LINE WITH CORRELATION OF LONG-RANGE IN THE DISORDER}

\author{
Edmundo Lazo ${ }^{1}$ \\ Recibido 19 de marzo de 2008, aceptado 3 de noviembre de 2008 \\ Received: March 19, 2008 Accepted: November 3, 2008
}

\begin{abstract}
RESUMEN
En este trabajo se estudia el comportamiento de las frecuencias permitidas en la línea de transmisión dual, cuando los valores de las capacitancias se distribuyen de acuerdo con una secuencia desordenada con correlación de largo alcance. Para cada frecuencia $\omega$ usamos el exponente Lyapunov $\lambda(\omega)$ como una herramienta para discriminar entre una línea de transmisión que deja pasar la señal y una que la filtra. Cuando la línea dual tiene una distribución periódica de capacitancias, aparece el típico esquema de bandas permitidas y prohibidas (gaps). Para el caso de una distribución al azar toda señal es filtrada. En este artículo reportamos el efecto del desorden con correlación de largo alcance. Entonces pueden aparecer dos casos dependiendo del tipo de correlación usado: a) sólo existe un número finito de frecuencias específicas para las cuales la señal se propaga, o b) existe una banda continua de frecuencias de propagación. La aparición de bandas continuas es un resultado muy importante, ya que la existencia de correlación en el desorden es una condición necesaria pero no suficiente para la aparición de frecuencias que se propagan. La comprensión de los mecanismos que describen la transición de una frecuencia prohibida a una frecuencia permitida, abre la posibilidad de fabricar dispositivos electrónicos donde dicha transición es relevante.
\end{abstract}

Palabras clave: Líneas de transmisión, desorden correlacionado, exponente de Lyapunov.

\begin{abstract}
In this work we study the behavior of the allowed frequencies in the dual transmission line when the values of capacitances are distributed according to a disordered sequence with long-range correlation. For each frequency $\omega$ we use the Lyapunov exponent $\lambda(\omega)$ as a tool to discriminate between a transmitting line and a filtering one. When the dual line has a periodic distribution of capacitances, a typical scheme of permitted bands and forbidden bands (gaps) appears. For the random case every signal is filtered. In this article we analyze the effect of long-range correlation, concluding that two cases can appear, depending on the kind of correlation used: a) there exist only a finite number of specific frequencies for which the signal is propagated, or $b$ ) there is a continuous band of propagating frequencies. The appearing of continuous bands is a very important result, since the existence of correlated disorder is a condition necessary but not sufficient to the appearing of propagating frequencies. Understanding the mechanisms that describe the transition from a forbidden frequency to an allowed frequency opens the possibility of manufacturing electronic devices where this transition is relevant.
\end{abstract}

Keywords: Transmission lines, correlated disorder, Lyapunov exponent.

\section{INTRODUCCIÓN}

El descubrimiento que los sistemas unidimensionales desordenados pueden tener transiciones metal-aislador [1-4] constituye un avance teórico crucial que tiene gran relevancia desde el punto de vista tecnológico, debido a que abre las posibilidades de fabricación de dispositivos con bordes de movilidad predeterminados, los cuales pueden ser usados como ventanas filtradoras en estructuras electrónicas, acústicas y fotónicas. En el mismo sentido, la comprensión de los mecanismos que describen la transición desde una frecuencia prohibida

\footnotetext{
1 Universidad de Tarapacá. Facultad de Ciencias. Departamento de Física. Arica, Chile. E-mail: elazo@uta.cl
} 
en una línea de transmisión a una frecuencia que permite el paso de la señal abre la posibilidad de llegar a fabricar dispositivos electrónicos donde dicha transición es relevante.

En términos teóricos, las propiedades de localización de modelos de estructura electrónica tipo enlace estrecho (tight-binding) fueron estudiadas inicialmente por Anderson [5], quien mostró que ciertos estados resultan ser localizados debido al desorden. La posterior generalización de este trabajo permitió probar que, en una dimensión, todos los estados son localizados para cualquier grado de desorden, siempre y cuando no exista correlación de corto o largo alcance en el desorden.

El estudio del grado de localización de la función de onda cuántica, en sistemas desordenados con correlación de corto y largo alcance en el desorden, ha sido objeto de gran atención en el último tiempo [1-15]. Recientemente se ha introducido correlación de largo alcance en el desorden a través del proceso de "dilución" de los sistemas desordenados [7-12]. Básicamente, la dilución consiste en introducir elementos no desordenados entre cada par de sitios desordenados. En términos generales, se obtiene un conjunto discreto de estados extendidos, cuyo número y posición energética dependen de la energía por sitio de los átomos que diluyen al sistema desordenado y del número de dichos átomos que se introducen entre dos sitios vecinos desordenados. En los sistemas diluidos no es posible encontrar bandas de estados extendidos.

En este trabajo se estudia la línea de transmisión dual. Esta línea es conocida como filtro paso alto, cuando se construye con un valor único de la capacitancia $C_{0}$ y un valor único de la inductancia $L_{0}$, en cualquier malla. En este caso, la línea presenta la máxima periodicidad ante la distribución de las capacitancias e inductancias.

En este trabajo estudiaremos el comportamiento de la línea de transmisión dual ante la presencia de diferentes tipos de desorden en la distribución de los valores de las capacitancias y las inductancias (desorden sustitucional). En general, el desorden sustitucional puede corresponder a una distribución al azar, sin ningún grado de correlación (tipo Anderson), o puede existir alguna correlación en la forma desordenada en que se distribuyen las capacitancias e inductancias en la línea de transmisión.
Sabemos que todos los sistemas unidimensionales con desorden sin correlación no presentan estados extendidos, o en el lenguaje de las líneas de transmisión ninguna señal se puede propagar a lo largo de la línea de transmisión cuando la distribución de inductancias y capacitancias es al azar. En general, en sistemas unidimensionales existen muy pocos casos en los cuales aparecen bandas de estados extendidos cuando existe correlación de largo alcance en el desorden [1-4,13,14]. Lo que ocurre es que la existencia de correlación en el desorden es condición necesaria, pero no suficiente, para la aparición de estados extendidos. A pesar de ello, lo que puede ocurrir en estos sistemas desordenados con correlación, tanto de corto alcance como de largo alcance, es la aparición de estados deslocalizados para algunas frecuencias de resonancia específicas $\omega_{r}$ [6-12]. Para correlación de corto alcance en el desorden, nunca se han encontrado bandas de estados extendidos.

\section{EL MODELO}

Consideremos la línea de transmisión dual desordenada, donde, en principio, las capacitancias $C_{j}$ y las inductancias $L_{j}$ pueden tomar valores distintos en cada celda, tal como lo muestra la figura 1.

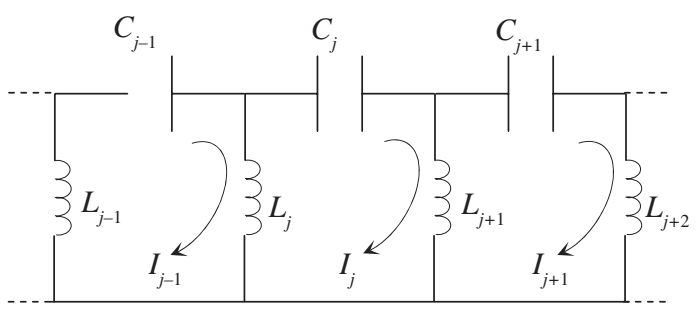

Figura 1. Línea tipo dual con desorden en todos sus parámetros.

La ecuación de movimiento de la celda j-ésima viene dada por:

$$
L_{j+1}\left(\dot{I}_{j}-\dot{I}_{j+1}\right)+L_{j}\left(\dot{I}_{j}-\dot{I}_{j-1}\right)=-\frac{q_{j}}{C_{j}}
$$

donde $I_{j}=\dot{q}_{j}$ y $\dot{I}_{j}=\ddot{q}_{j}$.

Consideremos los modos normales dados por:

$$
q_{j}=\phi_{j} e^{i \omega t}
$$


Reemplazando (2) en (1), se obtiene la ecuación dinámica para las amplitudes de los modos normales:

$$
\begin{array}{r}
\left(\frac{1}{\omega^{2} C_{j}}-L_{J}-L_{J+1}\right) \phi_{j}+L_{j} \phi_{j-1}+ \\
+L_{j+1} \phi_{j+1}=0
\end{array}
$$

Esta relación permite, en principio, obtener el comportamiento de las amplitudes $\phi_{j}$ en función de la frecuencia $\omega$. Para cada tipo de desorden, el valor absoluto del coeficiente del término $\phi_{j}$ en la relación (3) debe cumplir la siguiente condición:

$$
\frac{1}{L_{0}}\left|\frac{1}{\omega^{2} C_{j}}-L_{J}-L_{J+1}\right| \leq 2
$$

Donde $L_{0}$ es una unidad de inductancia. Esta relación impone restricciones sobre las frecuencias $\omega$ permitidas.

Si la línea de transmisión no presenta desorden en ninguno de los parámetros, es decir, si $C_{j}=C_{0}, \forall j$ y $L_{j}=L_{0}, \forall j$, se recupera el comportamiento del filtro paso alto, y la condición (4), queda:

$$
\left|\frac{1}{\omega^{2} C_{0} L_{0}}-2\right| \leq 2
$$

De esta expresión se obtiene la banda de frecuencias permitidas (filtro paso alto):

$$
\omega \geq \omega_{c}=\frac{1}{2 \sqrt{L_{0} C_{0}}}
$$

\section{RESULTADOS NUMÉRICOS}

Apliquemos ahora el formalismo del exponente de Lyapunov $\lambda(E)$ (ver Apéndice) [15] a la línea de transmisión dual. El exponente de Lyapunov $\lambda=\lambda(\omega)$ depende de la frecuencia $\omega[\mathrm{rad} / \mathrm{s}]$, y resulta ser una herramienta muy útil, pues permite discernir si la señal es filtrada o no a lo largo de la línea de transmisión, de acuerdo al siguiente criterio:

$$
\begin{aligned}
& \text { si } \lambda(\omega) \rightarrow 0 \rightarrow \text { la señal pasa } \\
& \text { si } \lambda(\omega)>0 \rightarrow \text { la señal es filtrada }
\end{aligned}
$$

En todo este trabajo, las inductancias tendrán siempre el mismo valor constante medido en Henry: $L_{j}=L_{0}=2.0[\mathrm{H}]$ en toda la línea de transmisión. Por lo tanto, la periodicidad y el desorden se referirán sólo a la secuencia de valores que tomarán las capacitancias $C_{j}$ en la línea de transmisión (desorden diagonal). Las capacitancias se medirán en Farad [F].

Para tener una visión completa del comportamiento de la línea dual estudiaremos dos casos muy diferentes: distribución periódica, y distribución desordenada (al azar y con correlación de largo alcance).

\section{Distribución periódica de $C_{j}$}

Caso puro: $C_{0} C_{0} C_{0} C_{0} C_{0} \ldots$

Consideremos en primer lugar el caso puro, es decir, el caso totalmente ordenado $C_{j}=C_{0} \forall j$. La figura 2 muestra $\lambda$ como función de $\omega$ para $C_{0}=1.0$

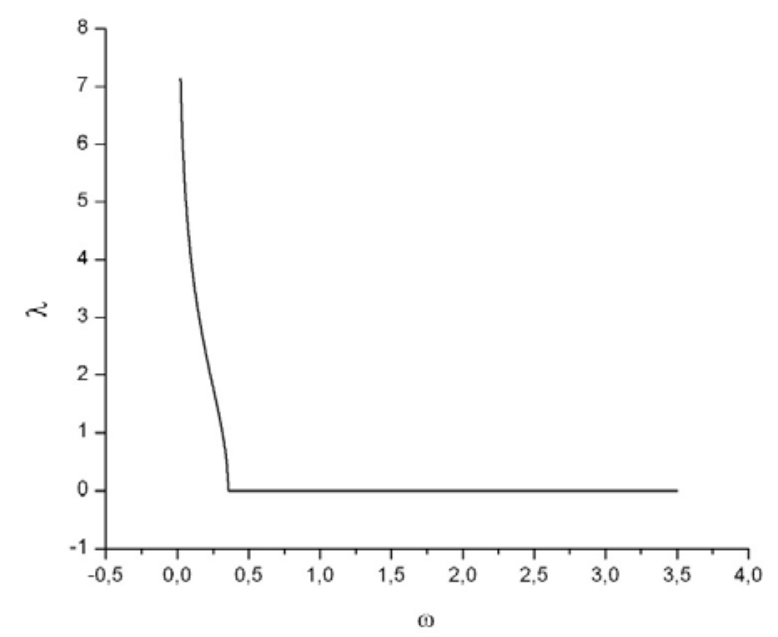

Figura 2. Filtro paso alto (línea de transmisión dual).

Allí puede verse que el exponente de Lyapunov tiende a cero, $\lambda \rightarrow 0$, para todo $\omega \geq \omega_{c}$, donde la frecuencia crítica $\omega_{c}$ para este caso vale $\omega_{c}=0.3535 \ldots$ Este resultado coincide con la frecuencia de corte dada por la relación (6). Por lo tanto, la línea presenta transmisión para frecuencias en el rango $\omega \geq \omega_{c}=0.3535 \ldots$ 
Caso periódico alternante: $C_{A} C_{B} C_{A} C_{B} C_{A} C_{B} \cdots$

En este caso distribuimos en forma alternada (distribución periódica) [16], dos valores distintos de capacitancia, $C_{A}$ y $C_{B}$. La introducción de esta periodicidad alternante hace aparecer bandas de frecuencias permitidas (rango de frecuencias $\omega$ donde $\lambda(\omega) \rightarrow 0$ ), separadas por bandas de frecuencias prohibidas o gap (rango de frecuencias $\omega$ donde $\lambda(\omega)>0$ ). La figura 3 muestra los resultados para el caso en que $C_{A}$ se mantiene fija, $C_{A}=0.5$, y $C_{B}$ toma cuatro valores distintos: $C_{B}=\{0.1,0.2,0.3,0.4\}$. Nótese que al crecer el valor de $C_{B}$ desde $C_{B}=0.1$ hasta $C_{B}=0.4$, el gap (banda de frecuencias prohibidas), asociado a valores $\lambda(\omega)>0$ se va haciendo más pequeño, mientras que el borde inferior de la banda permitida $(\lambda(\omega) \rightarrow 0)$ de menor frecuencia se va moviendo hacia la izquierda.

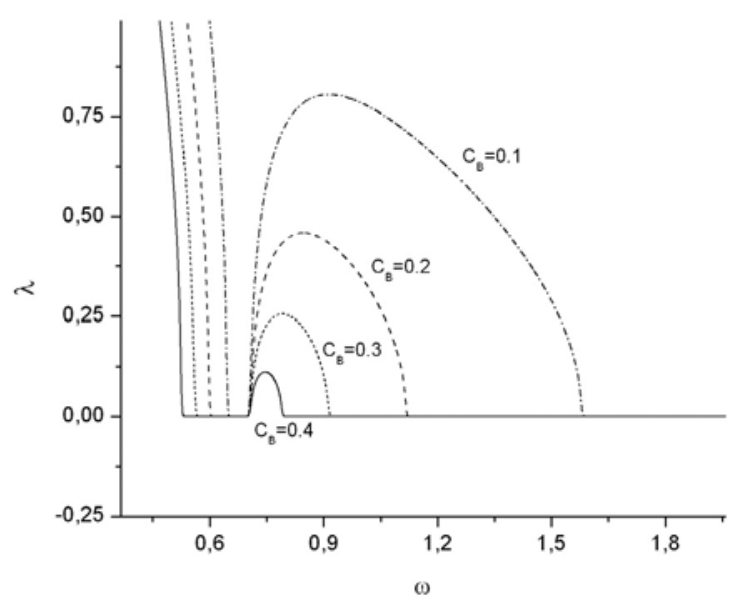

Figura 3. Línea dual con distribución alternante de capacitancias $C_{A}=0.5$, para distintos valores de $C_{B}=\{0.1,0.2,0.3,0.4\}$.

En el caso en que $C_{A}=C_{B}=0.5$, desaparece el gap de frecuencias prohibidas y se reobtiene el caso puro, cuya frecuencia de corte vale $\omega_{C}=0.5$

Caso superred: $C_{A} C_{A} C_{B} C_{B} C_{B} C_{A} C_{A} \cdots$

En este caso periódico distribuimos los $C_{j}$ en forma de una superred, formada por la repetición del siguiente patrón: el número de sitios con valor $C_{A}$ es $N_{A}=2$, y el número de sitios con valor $C_{B}$ es $N_{B}=3$

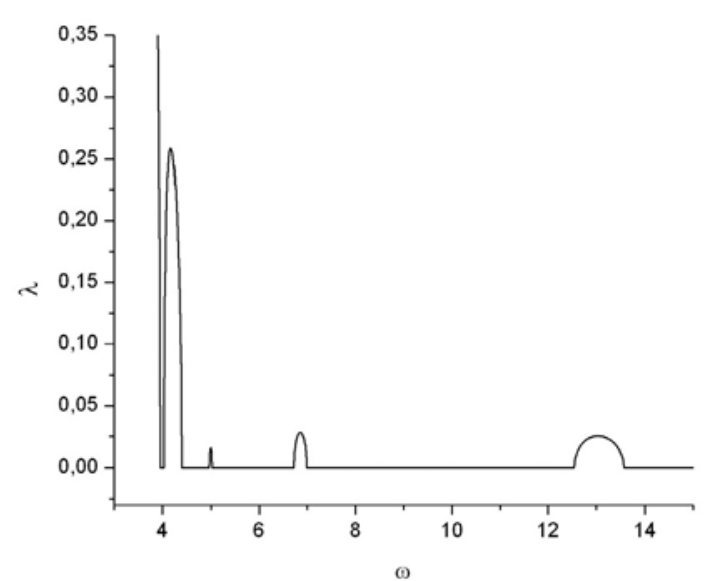

Figura 4. Superred de periodicidad $C_{A} C_{A} C_{B} C_{B} C_{B}$.

Para el cálculo numérico hemos usado los siguientes valores arbitrarios: $C_{A}=0.009$ y $C_{B}=0.007$, los cuales permiten visualizar mejor los efectos de formación de bandas de frecuencias permitidas y bandas de frecuencias prohibidas o gap, en las cuales la línea de transmisión filtra la señal. En este caso, dado que la periodicidad de la línea de transmisión es $p=N_{A}+N_{B}=5$, se espera la aparición de cinco bandas de frecuencias permitidas, separadas por gaps $(\lambda(\omega)>0)$. Las cinco bandas que permiten el paso de la frecuencia $(\lambda(\omega) \rightarrow 0)$ se ven claramente en la Fig. 4, aunque la banda de frecuencias permitida de la izquierda es muy estrecha.

\section{Distribución desordenada de $C_{j}$}

Caso al azar. $C_{j} \in(0,1)$

En este caso hacemos variar la capacitancia $C_{j}$ al azar, es decir, sin correlación, en el intervalo entre 0 y 1 , $C_{j} \in(0,1)$. 


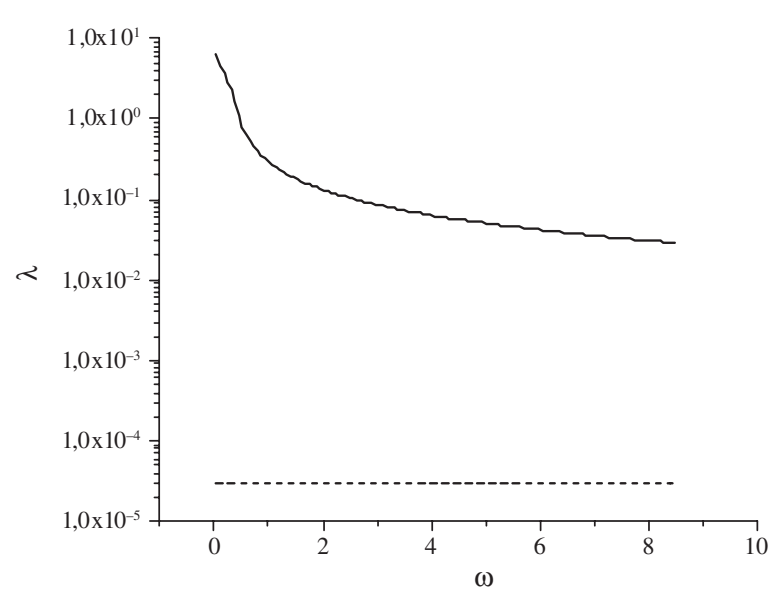

Figura 5. Línea dual con $C_{j}$ al azar. Nótese la escala vertical logarítmica.

La figura 5 muestra claramente que la línea de transmisión con desorden al azar o sin correlación filtra cualquier frecuencia, pues siempre se cumple que $\lambda(\omega)>\frac{1}{N}>0 \forall \omega$. Recordemos que de acuerdo a los criterios para sistemas de tamaño finito indicados en el Apéndice, cuando $\lambda(E)>\frac{1}{N}$, la línea de transmisión filtra la señal.

\section{Caso con desorden diluido}

En este caso estudiaremos un sistema desordenado con correlación de largo alcance mediante el llamado método de dilución. Consideremos una línea de transmisión en la cual $\operatorname{los} C_{j}$ se distribuyen según el siguiente procedimiento. Generamos primero una secuencia de valores de $C_{j}$ al azar, de modo que $C_{j} \in(0,1)$, luego, entre cada par de sitios desordenados introducimos un valor constante $C_{0}$, de manera que la cadena total queda "diluida" por los sitios con valor $C_{0}$. En este caso, la secuencia tendría la siguiente forma, si consideramos que los sitios con subíndice mayor que cero son desordenados:

$C_{1} C_{0} C_{3} C_{0} C_{5} C_{0} C_{7} C_{0} C_{9} C_{0} C_{11} \cdots$

Podemos generalizar este procedimiento y diluir la cadena desordenada al azar introduciendo entre dos sitios desordenados dos o más valores de $C_{0}$. Por ejemplo, si usamos tres valores de $C_{0}$ para diluir la línea desordenada, la secuencia quedaría:
$C_{1} C_{0} C_{0} C_{0} C_{5} C_{0} C_{0} C_{0} C_{9} C_{0} C_{0} C_{0} C_{13} \cdots$

En este sistema desordenado con correlación de largo alcance en el desorden no existen bandas de frecuencia permitida, pero sí es posible encontrar un conjunto discreto de frecuencias de resonancia $\omega_{r}$, que dejan pasar las señales. Dichas frecuencias permitidas se pueden obtener analíticamente, mediante un proceso de renormalización $[8,9]$ de las ecuaciones (3) que describen la dinámica de la amplitud de los modos normales. El formalismo de renormalización no está relacionado con el exponente de Lyapunov, el cual es sólo una herramienta que nos permite decidir, numéricamente, si una determinada frecuencia $\omega$ logrará cruzar por la línea de transmisión, o bien será filtrada por ella.

Las frecuencias $\omega_{r}$ que dejan pasar la señal cuando el sistema desordenado se diluye con $n$ capacitancias $C_{0}$, vienen dadas por las raíces de las ecuaciones polinomiales $D_{n}=0$, que vienen expresadas por la siguiente relación de recurrencia:

$$
D_{n}=D_{1} D_{n-1}-D_{n-2}
$$

con $n \geq 1$, y donde

$$
D_{0}=1, D_{1}=z_{0}=\left(\frac{1}{\omega^{2} L_{0} C_{0}}-2\right)
$$

Para los casos $n=1,2,3$, las raíces (frecuencias permitidas) son:

$n=1$

$$
\omega_{1}=\frac{1}{\sqrt{2 L_{0} C_{0}}}
$$

$n=2$

$$
\omega_{1}=\frac{1}{\sqrt{3 L_{0} C_{0}}}, \omega_{2}=\frac{1}{\sqrt{L_{0} C_{0}}}
$$


$n=3$

$$
\begin{aligned}
& \omega_{1}=\frac{1}{\sqrt{(2+\sqrt{2}) L_{0} C_{0}}}, \omega_{2}=\frac{1}{\sqrt{2 L_{0} C_{0}}}, \\
& \omega_{3}=\frac{1}{\sqrt{(2-\sqrt{2}) L_{0} C_{0}}}
\end{aligned}
$$

Para el cálculo numérico elegiremos el valor $C_{0}=1.0$ para diluir a la línea desordenada, manteniendo un único valor de la inductancia $L_{0}=2.0$. En ese caso, los resultados analíticos predicen las siguientes frecuencias de resonancia:

$$
\begin{aligned}
& n_{1}:\left\{\omega_{1}=0.5\right\} \\
& n_{2}:\left\{\omega_{1}=0.4083, \omega_{2}=0.7071\right\} \\
& n_{3}:\left\{\omega_{1}=0.3827, \omega_{2}=0.5, \omega_{3}=0.9239\right\}
\end{aligned}
$$

Consideremos como primer ejemplo, la dilución de la línea de transmisión usando un solo valor de $C_{0}$, de modo que en este caso los sitios pares son ordenados, $C_{2 j}=C_{0}$, y los sitios impares toman valores al azar, $C_{2 j-1}$ $\in(0,1)$. A pesar de que la secuencia de valores $C_{j}$ está diluida, debemos enfatizar que la secuencia completa, sigue siendo desordenada. De acuerdo al resultado analítico (10), y los datos numéricos, la única frecuencia permitida es $\omega_{1}=0.5$. La figura 6 muestra que la línea de transmisión diluida sigue siendo desordenada, ya que el exponente de Lyapunov es siempre mayor que cero $\left(\lambda(\omega)>\frac{1}{N}\right)$. Sin embargo, existe un único y específico valor de la frecuencia, $\omega_{r}=0.5$, para el cual $\lambda\left(\omega_{r}\right) \leq \frac{1}{N}$, permitiendo el paso de la señal a través de la línea de transmisión. Este resultado coincide completamente con el resultado predicho por (10) y (13). La línea punteada corresponde al valor $(1 / N)$.

La figura 7 muestra el detalle del comportamiento de $\lambda(\omega)$ en torno a $\omega_{r}=0.5$. Claramente se cumple $\lambda\left(\omega_{r}\right)<(1 / N)$ para $\omega_{r}=0.5$. De esta manera estamos completamente seguros que la línea de transmisión filtra todas las frecuencias pero deja pasar la señal justo cuando $\omega=\omega_{r}=0.5$.

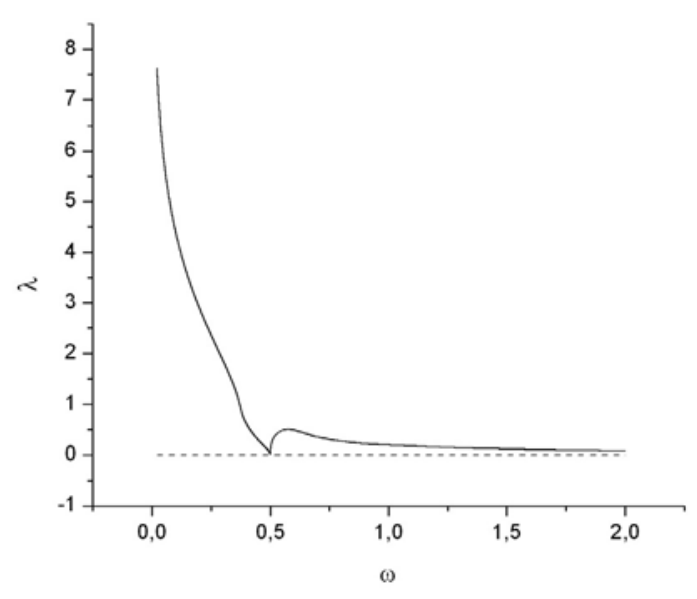

Figura 6. Cadena desordenada diluida con un solo valor de $C_{0}$. La línea punteada indica el valor $1 / N$.

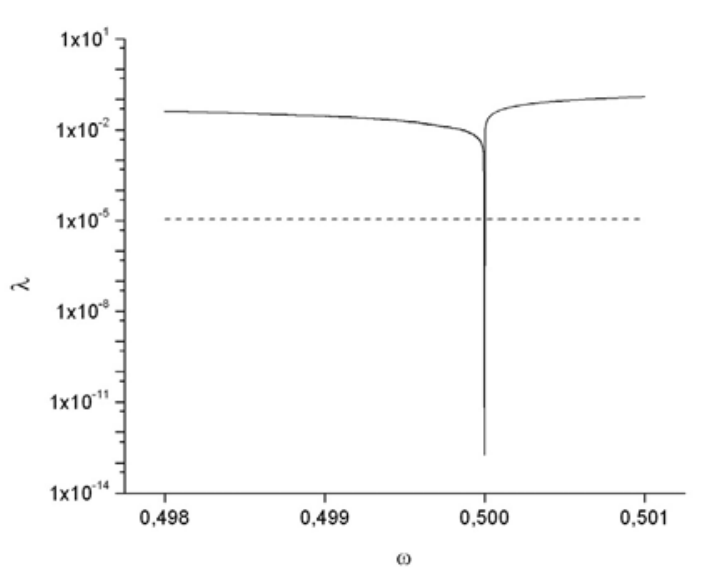

Figura 7. Detalle de la figura 6 en torno a $\omega_{r}=0.5$. La línea punteada indica el valor $1 / N$.

Como segundo ejemplo consideremos el caso en que la línea desordenada se diluye usando tres valores de $C_{0}$. La figura 8 muestra que el exponente de Lyapunov tiende a cero, $\lambda(\omega) \leq \frac{1}{N}$, solo para tres valores específicos y distintos de frecuencias: $\omega_{1}=0.384, \omega_{2}=0.5$ y $\omega_{3}=0.924$, y que para todo el resto de frecuencias, la línea filtra todas las señales. Este resultado coincide completamente con el resultado analítico obtenido en (13). 


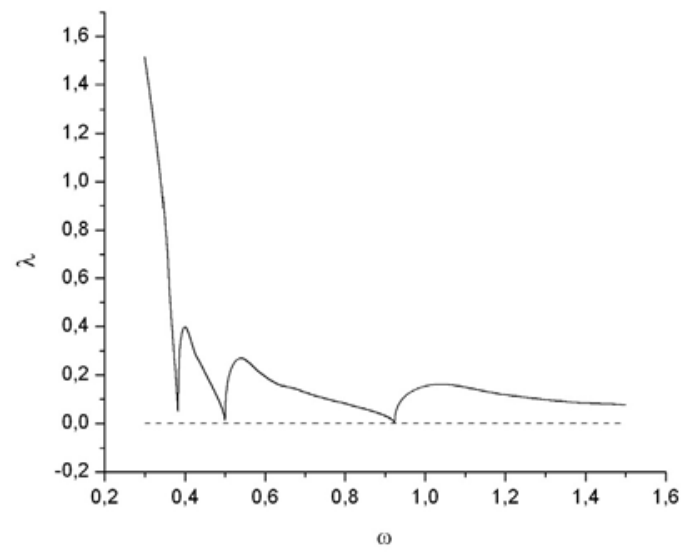

Figura 8. Cadena desordenada diluida con tres valores de $C_{0}$. La línea punteada indica el valor $1 / N$.

Dado que las frecuencias para las cuales se cumple la condición $\lambda(\omega) \leq \frac{1}{N}$ son muy específicas, no es fácil ver que la curva de la figura 8 llegue a cero, pero si hacemos un estudio en torno a los valores de los picos donde $\lambda(\omega) \rightarrow 0$, se descubre un comportamiento similar al mostrado en la figura 7 .

\section{Caso con parámetro de correlación de largo alcance $\alpha$}

Un caso de correlación de largo alcance, distinto al desorden diluido estudiado en el punto anterior, lo constituye la introducción de un sistema que considera tres capacitancias $C_{A}, C_{B}$ y $C_{C}$, distribuidas según una secuencia desordenada, pero que presenta correlación de largo alcance en el desorden. La secuencia de los tres valores de $\left\{C_{j}\right\}$ con correlación de largo alcance, se obtiene a través del método de filtrado de Fourier [17], que se describe a continuación. En primer lugar, se genera una secuencia de números al azar $\left\{u_{j}\right\}$, es decir, sin correlación espacial. Luego se calcula la transformada directa de Fourier de los $\left\{u_{j}\right\}$, obteniéndose la secuencia $\left\{u_{k}\right\}$. A partir de esa secuencia, se genera la secuencia $\left\{v_{k}\right\}$, a través del siguiente proceso:

$$
v_{k}=k^{-(2 \alpha-1) / 2} u_{k}
$$

Finalmente se calcula la transformada inversa de Fourier de la secuencia $\left\{v_{k}\right\}$, obteniéndose la secuencia $\left\{v_{j}\right\}$.
Esta secuencia resultante tiene una correlación espacial con densidad espectral $S(k)$ dada por

$$
S(k) \propto k^{-(2 \alpha-1)}
$$

El exponente $\alpha$ se denomina exponente de correlación y cuantifica el grado de correlación de largo alcance que contiene la secuencia. Nótese que el caso $\alpha=0.5$ corresponde a una secuencia con desorden no correlacionado $\mathrm{o}$ al azar, también llamado ruido blanco. El caso $\alpha>0.5$ indica una correlación positiva.

Una vez que las secuencias $\left\{v_{j}\right\}$ son generadas usando el método de filtrado de Fourier, realizamos un proceso de normalización, de modo que el promedio valga cero, $\left\langle v_{j}\right\rangle=0$, y que la variancia valga 1 , esto es, $\sigma^{2}=\left\langle v_{j}^{2}\right\rangle-\left\langle v_{j}\right\rangle^{2}=1$.

A partir de la secuencia con correlación de largo alcance $\left\{v_{j}\right\}$, generamos la distribución de tres valores de capacitancias $C_{A}, C_{B}$ y $C_{0}$, según el siguiente mapeo [13]:

$$
C_{j}=\left\{\begin{array}{cc}
C_{A} & v_{j}<-a \\
C_{0} & -a \leq v_{j} \leq a \\
C_{B} & v_{j}>a
\end{array}\right.
$$

Donde $a$ es un parámetro que determina la concentración de los sitios con capacitancia $C_{0}$.

El estudio numérico del comportamiento del exponente de Lyapunov, $\lambda(\omega)$, en función del exponente de correlación $\alpha$, muestra que para cada $\alpha \geq 0.8$, siempre existe un valor del parámetro $a$ para el cual comienzan a aparecer bandas continuas de frecuencias permitidas, es decir, aparecen rangos de valores de frecuencia $\omega$ para los cuales la línea de transmisión deja pasar la señal. La aparición de bandas de frecuencias permitidas es un resultado muy importante en el contexto de sistemas unidimensionales desordenados con correlación.

Como ejemplo de la situación descrita, la figura 9 muestra el exponente de Lyapunov para diferentes valores del parámetro $a$, para el valor $\alpha=1.0$. En este caso hemos usado los siguientes valores de capacitancias 
en [Farad]: $C_{A}=0.1, C_{B}=0.3$ y $C_{0}=0.5$. Se usaron líneas de transmisión de largo $N=2^{15}$ y el exponente de Lyapunov se ha obtenido después de promediar sobre 500 distribuciones con desorden correlacionado. Adicionalmente, para disminuir la fluctuación numérica se ha graficado el promedio del exponente de Lyapunov correspondiente al promedio de varias energías muy cercanas.

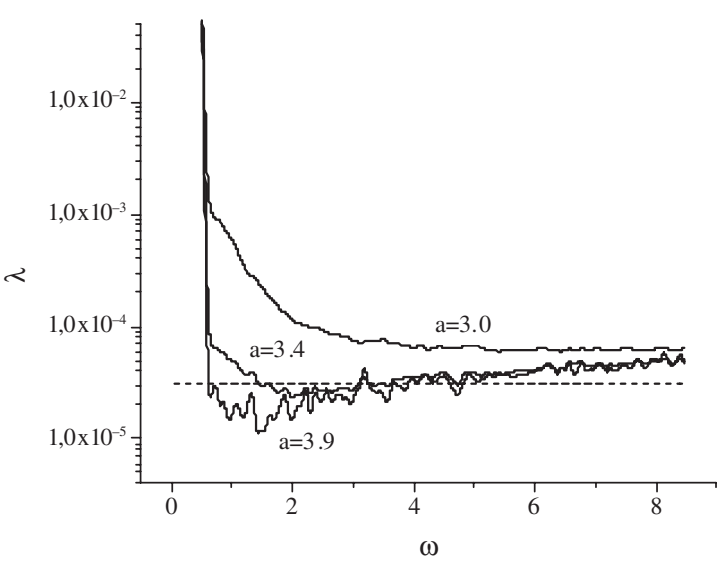

Figura 9. Exponente de Lyapunov en función de la frecuencia para tres valores del parámetro $a$, para $\alpha=1.0$. La línea punteada marca el valor $1 / N$.

Claramente se ve que existen bandas continuas de valores de frecuencia $\omega$ para las cuales se cumple que $\lambda(\omega) \leq \frac{1}{N}$, para dos valores distintos del parámetro $a: a=3.4$ y $a=3.9$. Por lo tanto, para estas bandas continuas de frecuencia, la línea de transmisión deja pasar las señales. Sin embargo, para $a=3.0$ la línea filtra todas las frecuencias, ya que se cumple que $\lambda(\omega)>\frac{1}{N}, \forall \omega$

Para valores de $a>3.9$ la banda de frecuencias permitidas sigue creciendo, debido a que cada vez hay menos desorden en la línea de transmisión, o dicho de otro modo, si $a$ es muy grande, casi toda la secuencia de valores de las capacitancias son del tipo $C_{0}$, ya que estos valores $C_{0}$ aparecen cuando $-a \leq v_{j} \leq a$. Nótese también que al crecer $a$, el borde izquierdo de la banda permitida se mueve hacia la izquierda. Este desplazamiento se detiene cuando la línea de transmisión contiene solo capacitancias $C_{0}$, ya que entonces se trata de un filtro paso alto, donde existe una única banda de valores permitidos para $\omega \geq \omega_{c}=0.5$, para los valores $C_{0}=0.5[\mathrm{~F}]$ y $L_{0}=2.0[\mathrm{H}]$ que estamos empleando en el cálculo numérico.

\section{RESUMEN Y CONCLUSIONES}

En este trabajo hemos estudiado la línea de transmisión dual con distribución periódica y desordenada de capacitancias, usando el exponente de Lyapunov $\lambda(\omega)$ como herramienta para determinar si la línea de transmisión es capaz de dejar pasar o de filtrar una señal de determinada frecuencia, ante un desorden determinado en la distribución de capacitancias.

Usando una analogía con la mecánica cuántica, hemos determinado que si el exponente de Lyapunov tiende a cero, $\lambda(\omega) \rightarrow 0$, la línea de transmisión deja pasar la señal, pero si el exponente de Lyapunov es mayor que cero, $\lambda(\omega)>0$, la línea de transmisión impide o filtra el paso de la señal. Este criterio se modifica para líneas de transmisión de tamaño finito $N$, de modo que si $\lambda(\omega) \leq \frac{1}{N}$ la línea de transmisión deja pasar la señal, pero si $\lambda\left(\omega_{r}\right)>\frac{1}{N}$, la línea de transmisión impide o filtra el paso de la señal.

Numéricamente, hemos demostrado que para líneas de transmisión periódicas existen bandas de frecuencia para las cuales la señal pasa a través de la línea (bandas permitidas), y hemos encontrado bandas de frecuencia, para las cuales la línea filtra la señal (bandas prohibidas o gap).

Para el caso de líneas de transmisión desordenadas al azar (sin correlación), la línea filtra todas las frecuencias. Para el caso de desorden diluido (un tipo de correlación de largo alcance), la línea filtra todas las frecuencias, a excepción de un conjunto finito de frecuencias de resonancia muy especiales $\omega_{r}$, para las cuales la línea de transmisión deja pasar la señal. Para el caso de desorden de largo alcance generado por el método de filtrado de Fourier y controlado por el exponente de correlación $\alpha$, hemos encontrado bandas continuas de frecuencias permitidas para las cuales se produce la transmisión de señales a través de la línea de transmisión. Estas bandas de frecuencia permitidas son muy importantes ya que pueden ser encontradas experimentalmente, a diferencia 
de las frecuencias de resonancia $\omega_{r}$ de más difícil ubicación experimental. El resultado más importante de este trabajo es que hemos demostrado numéricamente que la correlación de largo alcance en el desorden produce bandas de frecuencias permitidas a través de la línea de transmisión dual. Esto no es un hecho trivial, ya que sabemos que la existencia de correlación en el desorden es una condición necesaria, pero no suficiente para la aparición de bandas de frecuencias permitidas. Otro resultado importante es la aplicación de técnicas de la mecánica cuántica en una línea de transmisión clásica desordenada. Específicamente, hemos demostrado que el exponente de Lyapunov es una herramienta muy efectiva para determinar la capacidad de conducir señales en una línea de transmisión. Finalmente, queremos hacer notar que a pesar de que los cálculos numéricos fueron hechos considerando un número finito de celdas en la línea de transmisión, $\mathrm{N}=2^{15}=32.768$, los resultados obtenidos para valores pequeños de $N$ son básicamente los mismos, notándose que las bandas de conducción son un poco más anchas para valores pequeños de $N$ para un mismo valor del exponente de correlación $\alpha$. Por otra parte, es interesante hacer notar que la falta de periodicidad, introducida al azar en la fabricación de líneas de transmisión, resulta ser apenas un pequeño ruido en una red finita de tamaño razonable y, por lo tanto, no cambia los comportamientos esenciales de dicha red finita.

\section{APÉNDICE}

\section{Analogía cuántica}

En este trabajo hemos utilizado la línea de transmisión dual para estudiar el efecto del desorden, con y sin correlación, sobre el espectro de valores de frecuencia $\omega$ permitidos. En este modelo hemos encontrado una relación entre las amplitudes de los modos normales, del tipo general (ver relación (3)):

$$
z_{j} \phi_{j}+v_{j-1} \phi_{j-1}+v_{j+1} \phi_{j+1}=0
$$

El término diagonal $z_{j}$ es función de la frecuencia $\omega$ y de los parámetros del sistema $z_{j}=z_{j}\left(\omega, C_{j}, L_{j}\right)$. Los términos no diagonales, $v_{j \pm 1}$ sólo dependen de $C_{j}$ y $L_{j}$, es decir, $v_{j \pm 1}=v_{j \pm 1}\left(C_{j}, L_{j}\right)$.
La línea de transmisión dual deja pasar señales de cierta frecuencia, pero impide el paso de señales de otras frecuencias, incluso en el caso de desorden correlacionado. Este comportamiento de las señales eléctricas en las líneas de transmisión se puede caracterizar usando un procedimiento análogo al empleado en mecánica cuántica, al describir el grado de localización o de deslocalización (extensión espacial) de las funciones de onda cuántica que describen el comportamiento de un electrón en un sistema desordenado. Para mostrar esta analogía, estudiaremos un sistema unidimensional muy simple.

Consideremos un electrón moviéndose en una red unidimensional, la cual está formada por una cadena lineal con energías por sitio $\varepsilon_{j}$ y probabilidades de salto a vecinos más cercanos de un sitio a otro de la cadena $v_{j, j \pm 1}=v_{j \pm 1, j}$, tal como lo muestra la figura 10.

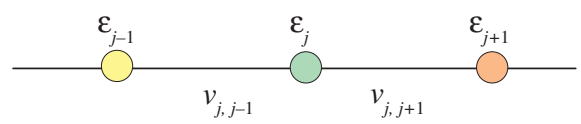

Figura 10. Parte de una cadena lineal desordenada.

La ecuación de Schröedinger independiente del tiempo viene dada por,

$$
\hat{H} \psi=E \psi
$$

Donde $\hat{H}$ representa el hamiltoniano que describe al electrón con energía $E$ en la red, y $\psi$ es la función de onda cuántica, la cual se puede escribir en la forma:

$$
\psi=\sum_{j=1}^{N} \phi_{j}|j\rangle
$$

donde $\phi_{j}$ representa la amplitud de la función de onda en cada sitio $j$ de la cadena. Conocido el hamiltoniano, y reemplazando (19) en (18), se tiene la ecuación dinámica para el electrón en la cadena, la cual toma la forma genérica:

$$
z_{j} \phi_{j}+v_{j-1} \phi_{j-1}+v_{j+1} \phi_{j+1}=0
$$

Esta ecuación tiene la misma forma funcional que la relación (3) que describe a la línea de transmisión dual. El término diagonal de la relación (17) es función de la 
energía $E$ y de los parámetros $\varepsilon_{j}$ y $v_{j, j \pm 1}$ del sistema, es decir, $z_{j}=z_{j}\left(E, \varepsilon_{j}, v_{j, j \pm 1}\right)$.

La caracterización de los estados cuánticos viene descrita por el llamado exponente de Lyapunov $\lambda(E)$, el cual depende de la energía $E$ del electrón y depende además, del grado y tipo de desorden de la cadena en la cual el electrón se va a propagar. El exponente de Lyapunov $\lambda(E)$ se define de la siguiente manera:

$$
\lambda(E)=\lim _{N \rightarrow \infty} \frac{1}{N} \sum_{j=1}^{N} \ln \left|\frac{\phi_{j+1}}{\phi_{j}}\right|
$$

El cociente $\left(\frac{\phi_{j+1}}{\phi_{j}}\right)$ se puede calcular en forma recursiva a partir de los valores de la energía $E$ y de los parámetros $z_{j}$ y $v_{j, j \pm 1}$ que aparecen en la relación (20).

Los valores que asume el exponente de Lyapunov permiten clasificar a los estados cuánticos como extendidos o localizados, es decir, permiten caracterizar el grado de localización de la función de onda $\psi$, en la siguiente forma:

$$
\begin{array}{ll}
\text { si } \lambda(E) \rightarrow 0 & \text { función extendida } \\
\text { si } \lambda(E)>0 & \text { función localizada }
\end{array}
$$

\section{Función de onda extendida o deslocalizada}

Si $\lambda(E) \rightarrow 0$, entonces las amplitudes $\phi_{j}$ de la función de onda definidas en la relación (19), son distintas de cero en todos los puntos de la cadena, lo cual implica que existe una probabilidad distinta de cero de hallar al electrón en cualquier sitio de la red. Todo esto caracteriza a una función de onda extendida en el espacio o función de onda deslocalizada.

\section{Función de onda localizada}

Si $\lambda(E)>0$, la función de onda está localizada en una pequeña región de la cadena, lo cual implica que las amplitudes $\phi_{j}$ de la función de onda son distintas de cero sólo en algunos puntos de la cadena.
Existe una relación entre el coeficiente de transmisión $T(E)$ de un lado al otro de la cadena de $N$ sitios, y el exponente de Lyapunov $\lambda(E)$, dada por

$$
T(E)=e^{-N \lambda(E)}
$$

Nótese que si $\lambda(E) \rightarrow 0$ (función de onda extendida), el coeficiente de transmisión tiende al valor $1, T(E) \rightarrow 1$, es decir, la transmisión es perfecta. $\mathrm{Si}$, por el contrario, $\lambda(E)>0$ (función de onda localizada), el coeficiente de transmisión tiende a cero en la forma, $T(E)=\frac{1}{e^{N \lambda(E)}} \rightarrow 0$, y por lo tanto, no hay transmisión de señal de un punto a otro de la cadena.

Otro parámetro útil para describir el grado de localización de la función de onda, es la llamada longitud de localización $\Lambda$, definida como el inverso del exponente de Lyapunov $\lambda$,

$$
\Lambda(E)=\frac{1}{\lambda(E)}
$$

Si la función de onda es extendida, $\lambda(E) \rightarrow 0$, esto implica que la longitud de localización tiende a infinito, $\Lambda(E) \rightarrow \infty$. Si la función de onda es localizada, $\lambda(E)>0$, la longitud de localización es finita.

En la práctica, cuando estudiamos sistemas con un número finito de sitios $N$, podemos decir que un estado es extendido si la longitud de localización $\Lambda$ es mayor o igual que el tamaño $N$ del sistema, $\Lambda \geq N$. Usando la relación (24), escribimos,

$$
\Lambda(E)=\frac{1}{\lambda(E)} \geq N
$$

Por lo tanto, podemos decir que un estado es extendido, si cumple con la condición,

$$
\lambda(E) \leq \frac{1}{N}
$$


En cambio, para estados localizados, $\lambda(E)>0$, la longitud de localización $\Lambda$ es finita y menor que el largo de la cadena $N$, es decir, $\Lambda<N$. Usando (24), escribimos,

$$
\lambda(E)>\frac{1}{N}
$$

\section{AGRADECIMIENTOS}

E. Lazo agradece el financiamiento para la ejecución de este trabajo al Proyecto de investigación UTA $\mathrm{N}^{\circ} 4720-07$.

\section{REFERENCIAS}

[1] V. Bellani, E. Diez, R. Hey, L. Tony, L. Tarricone, G. B. Parravicini, F. Domínguez-Adame and R. Gómez-Alcala. "Experimental evidence of delocalized states in random dimmer superlattices". Phys. Rev. Lett. Vol. 82, p. 2159. 1999.

[2] F.A.B.F. de Moura and M.L. Lyra. "Delocalization in the 1D Anderson Model with long-Range Correlated Disorder". Phys. Rev. Lett. Vol. 81, pp. 3735-3738. 1998. "Correlation-induced metal-insulator transition in the 1D Anderson model", Physica A. Vol. $266 \mathrm{~N}^{\circ}$ 1, pp. 465-470 (6). 1999.

[3] F.M. Izrailev and A.A. Krokhin. "Localization and the mobility edge in one-dimensional potentials with correlated disorder". Phys. Rev. Lett. Vol. 82, pp. 4062-4065. 1999. F.M. Izrailev, A.A. Krokhin, S.E. Ulloa. "Mobility edge in aperiodic Kronig-Penney potentials with correlated disorder: perturbative approach". Phys. Rev. B. Vol. 63, pp. 041102.1-041102.4. 2001.

[4] P. Carpena, P.B. Galvan, P. Ch. Ivanov and H.E. Stanley. "Metal insulator transition in chains with correlated disorder". Nature. Vol. 418, pp. 955-959. 2002.

[5] P.W. Anderson. "Absence of diffusion in certain random lattices". Phys. Rev. Vol. 109, pp. 14921505. 1958.
[6] D.H. Dunlap, H.L. Wu, P.W. Phillips. "Absence of localization in a random-dimmer model". Phys. Rev. Lett. Vol. 65, pp. 88-91. 1990.

[7] M. Hilke. "Localization properties of the periodic random Anderson model". J. Phys. A: Math. Gen. Vol. 30, pp. L367-L371. 1997.

[8] E. Lazo, M.E. Onell. "Existence of delocalized states in two interpenetrated 1-D diluted Anderson chains". Phys. Lett. A. Vol. 283, pp. 376-381. 2001.

[9] E. Lazo, M.E. Onell. "Extended states in 1-D Anderson chain diluted by periodic disorder". Physica B. Vol. 299, pp. 173-179. 2001.

[10] F.A.B.F. de Moura, M.N.B. Santos, U.L. Fulco, M.L. Lyra, E. Lazo, M.E. Onell. "Delocalization and density of states in one-dimensional diluted Anderson model". Eur. Phys. J. B Vol. 36, pp. 81-84. 2003.

[11] E.L. Albuquerque, M.S. Vasconcelos, M.L. Lyra, F.A.B.F. de Moura. "Nucleotide correlations and electronic transport of DNA sequences". Phys. Rev. E. Vol. 71, pp. 021910.1-021910.7. 2005.

[12] S.S. Albuquerque, F.A.B.F. de Moura, M.L. Lyra, E. Lazo. "Sensitivity to initial conditions of wave-packet dynamics in the dilute Anderson chain". Physics Letters A. Vol. 355, pp. 468-472. 2006.

[13] A. Esmailpour, M. Esmailzadeh, E. Faizabadi, P. Carpena, M. Reza Rahimi Tabar. "Metal-insulator transition in random Kronig-Penney superlattices with long-range correlated disorder". Phys. Rev. B. Vol. 74, pp. 024206-024212. 2006.

[14] A. Esmailpour, H. Cheraghchi, Pedro Carpena, M. Reza Rahimi Tabar. "Metal-Insulator transition in a ternary model with long range correlated disorder”. J. Stat. Mech, pp. 09014. 2007.

[15] J. Roessler, G. Martínez, M. Kiwi. "Random chains and complex transfer matrix attractor". Solid State Comm. Vol. 61, pp. 395-400. 1987. 
[16] J. Roessler, E. Lazo. "A generalization of the locator coherent-potential approximation for alloys with shor-range order". J. Phys. C: Solid State Phys. Vol. 14, pp. 3499. 1981.
[17] H.A. Makse, S. Havlin, M. Schwartz and H.E. Stanley. "Methods for generating long-range correlations for large systems". Phys. E. Vol. 53, pp. 5445-5449. 1996. 